\title{
Análise cefalométrica comparativa das alturas faciais, anterior e posterior, em jovens brasileiros, descendentes de xantodermas e leucodermas, com oclusão normal
}

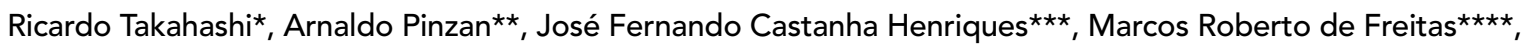

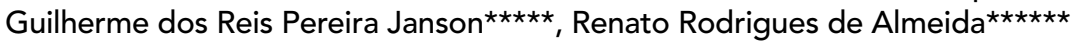

\section{Resumo}

Objetivos: avaliou-se cefalometricamente as estruturas esqueléticas da face no sentido crâniocaudal (vertical) para: obter os valores médios de normalidade de jovens brasileiros, descendentes de xantodermas e leucodermas, com oclusão normal; verificar a presença ou ausência de dimorfismo entre os gêneros e investigar a existência de diferenças ou semelhanças entre os grupos raciais estudados. Metodologia: estudo cefalométrico. Resultados: após a obtenção da média e do desvio padrão de cada medida cefalométrica estudada, para ambos os gêneros e grupos raciais, os resultados sinalizaram, em algumas das grandezas cefalométricas, a presença de dimorfismo entre os gêneros para ambos os grupos raciais e diferenças entre os grupos raciais. Conclusões: verificou-se a necessidade de se utilizar um padrão cefalométrico específico, no sentido crânio-caudal (vertical), para os brasileiros, descendentes de xantodermas e leucodermas.

Palavras-chave: Padrões cefalométricos. Oclusão normal. Dimensão vertical.

Doutor em Odontologia, área Ortodontia (FOB - USP), Coordenador do Curso de Especialização em Ortodontia da UNIPAR.

** Professor Associado do Departamento de Ortodontia, Odontopediatria e Saúde Coletiva da FOB-USP, Professor da Disciplina de Ortodontia da USC e Professor da UNICID.

*** Professor Titular do Departamento de Ortodontia, Odontopediatria e Saúde Coletiva da FOB-USP e Coordenador do Curso de Pós-graduação ao nível de Doutorado da Disciplina de Ortodontia da FOB-USP.

**** Professor Associado do Departamento de Ortodontia, Odontopediatria e Saúde Coletiva da FOB-USP e Chefe do Departamento de Ortodontia da FOB-USP.

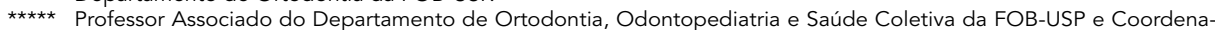
dor do Curso de Pós-graduação ao nível de Mestrado da Disciplina de Ortodontia da FOB-USP.

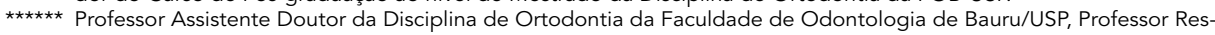
ponsável pela Disciplina de Ortodontia da Faculdade de Odontologia de Lins, Coordenador do Curso de Especialização em Ortodontia da F.O.L./UNIMEP e Professor Titular da UNICID. 
A preocupação em se diagnosticar, planejar e conseguir correções ortodônticas estáveis com o correto posicionamento dos dentes, resultando na harmonia facial e beleza no sorriso dos pacientes, tem sido constante, desde os primórdios da Ortodontia ${ }^{2}$.

Os elementos de diagnóstico e a evolução na tecnologia dos materiais oferecem, a cada dia, melhores condições de se realizar um correto diagnóstico, plano de tratamento, correção das más oclusões e estabilidade dos casos. Dentre os elementos de diagnóstico, as telerradiografias cefalométricas, em norma lateral, constituem um importante auxílio na determinação dos possíveis problemas esqueléticos, dentários e tegumentares de indivíduos com má oclusão.

A partir destas radiografias, pode-se analisar a face nos sentidos ântero-posterior ${ }^{16,17}$, $18,19,65,76,77,78,79,84$ e crânio-caudal (vertical) ${ }^{24,32,49 \text {, }}$ $51,66,70,71,74,87,88,89,90,91,92,93,94$, por meio das análises cefalométricas. A análise no sentido ântero-posterior da face tem sido mais utilizada; entretanto, o diagnóstico das estruturas craniofaciais, no sentido crânio-caudal (vertical), possui grande importância e deve ser considerado nas diferentes fases do tratamento ortodôntico, pelo fato de um desequilíbrio, no sentido crânio-caudal (vertical) da face, poder proporcionar uma alteração significante no sentido ântero-posterior.

Os componentes crânio-caudais (verticais) da face inicialmente foram pesquisados no intuito de propiciar o restabelecimento dentário com próteses dentárias, permitindo a reconstrução da harmonia facial ${ }^{31}$. Observou-se que a variação na dimensão crânio-caudal (vertical) poderia influenciar, principalmente, na posição mandibular. A partir desta observação, começaram a surgir diferentes análises cefalométricas que examinavam a face no sentido crânio-caudal (vertical). ${ }^{6,49,70,71,89,93,94}$

Estudos do crescimento da face $7,8,9,10,11,12,13,14$, $15,20,21,22,29,33,34,35,36,37,39,42,44$, no sentido crânio-caudal (vertical), evidenciaram que a desarmonia entre os componentes crânio-caudais (verticais) e ânte- ro-posteriores das estruturas craniofaciais podem possibilitar a rotação mandibular, no sentido horário ou anti-horário 3,5,28,45,52,56,57,60,62,63, 64,67,68,70,71, 72 $, 73,80,81,82,83$, podendo contribuir com o desenvolvimento da sobremordida profunda ou da mordida aberta anterior ${ }^{58,59}$. No entanto, o tratamento ortodôntico ${ }^{30}$ também pode proporcionar o equilíbrio ou desequilíbrio na face, causando mudanças desejáveis ou indesejáveis. . $^{4,423,25,26,27,46,48,61,69,75,85,86}$

A análise crânio-caudal (vertical) da face, por meio das alturas faciais anteriores e posteriores, pelas proporções faciais e pelos índices faciais, proporciona um exame preciso das discrepâncias crânio-caudais (verticais) da face $38,40,41,43,47,50,53,54,55$, bem como o monitoramento da dimensão crâniocaudal (vertical), nos diferentes estágios do tratamento ortodôntico, a fim de contribuir na obtenção da harmonia e do equilíbrio facial, da saúde dos tecidos, da eficiência mastigatória, da proximidade de uma oclusão normal e da estabilidade dos resultados ortodônticos.

A presente pesquisa possui o propósito de estudar cefalometricamente as estruturas esqueléticas da face no sentido crânio-caudal (vertical) para: obter os valores médios de normalidade de jovens brasileiros, descendentes de xantodermas e leucodermas, com oclusão normal; verificar a presença ou ausência de dimorfismo entre os gêneros e investigar a existência de diferenças ou semelhanças entre os grupos raciais estudados.

\section{MATERIAL E MÉTODOS}

O material empregado, na realização deste trabalho, constou de 118 telerradiografias cefalométricas, em norma lateral, oriundas de amostras de brasileiros xantodermas japoneses e leucodermas com oclusão normal, pertencentes ao Departamento de Odontopediatria, Ortodontia e Saúde Coletiva da Faculdade de Odontologia de Bauru da Universidade de São Paulo.

A amostra xantoderma foi composta por 40 jovens, sendo 20 do gênero feminino e 20 do masculino, descendentes de japoneses, com oclusão 
normal, sem ter sido submetidos a tratamento ortodôntico, sem mutilações e idade média de 15,71 anos. A leucoderma constitui-se de 78 jovens, sendo 39 do gênero feminino e 39 do masculino, descendentes de espanhóis, italianos e portugueses, com oclusão normal, sem ter sido submetidos a tratamento ortodôntico, sem mutilações e idade média de 13,5 anos.

Os integrantes da pesquisa possuíam todos os dentes permanentes em oclusão, com ausência dos terceiros molares, relações normais de molares, pequeno ou nenhum grau de apinhamento, ausência de mordida cruzada, trespasses vertical e horizontal normais, ausência de diferenças entre as posições mandibulares de Relação Cêntrica e Máxima Intercuspidação Habitual e perfis agradáveis.

\section{Métodos}

As telerradiografias cefalométricas das amostras de oclusão normal de xantodermas e leucodermas, pertencentes ao Departamento de Odontopediatria, Ortodontia e Saúde Coletiva da Faculdade de Odontologia de Bauru, Universidade de São Paulo, foram obtidas e reveladas de acordo com as normas preconizadas pela Disciplina de Radiologia da mesma instituição. $\mathrm{O}$ fator de magnificação da imagem radiográfica de cada aparelho utilizado foi calculado e posteriormente efetuou-se a correção deste fator para que, durante a comparação entre as amostras, se obtivesse maior precisão.

Após a obtenção das telerradiografias cefalométricas, em norma lateral, realizou-se o desenho anatômico, a localização dos pontos cefalométricos clássicos, o traçado das linhas e planos e a demarcação dos pontos construídos, Ar' e ENA', de acordo com Wylie, Johnson ${ }^{94}$; Siriwat, Jarabak ${ }^{74}$; Horn $^{32}$; Gebeck ${ }^{24}$ e Merrifield ${ }^{51}$ (Fig. 1, 2, 3, 4).

\section{Análise descritiva e comparativa da amostra de xantodermas e leucodermas}

Com o propósito de descrever as amostras xantoderma e leucoderma, foram utilizados médias e desvios padrão. Para verificar a existência de dimorfismo entre gêneros, aplicou-se o teste $t$ de Student ao nível de 5\% de significância para as duas amostras.

No intuito de comparar as medidas analisadas, em xantodermas e leucodermas, de ambos os gêneros, foram calculadas as diferenças entre médias de cada medida para cada gênero e feitas as diferenças, entre xantodermas e leucodermas. Depois foi aplicado o teste $t$ de Student, ao nível de significância de $5 \%$, para as diferenças obtidas.

\section{RESULTADOS}

Os dados, referentes aos valores individuais dos integrantes das amostras xantoderma e leucoderma, foram submetidos à análise estatística. Primeiro, foram calculados a média e o desvio padrão de cada medida cefalométrica estudada, para ambos os gêneros e para as duas raças. Estes resultados estão nas tabelas 1, 2, 3 e 4 .

Com base nos resultados apresentados nessas tabelas, foi procurada a presença de dimorfismo entre gêneros, tanto em xantodermas como leucodermas. Para isso, foram calculadas as diferenças entre médias de gêneros, para cada medida, tanto para xantodermas como leucodermas e, depois, foi aplicado o teste $t$ de Student, para diferença obtida. Os resultados estão na tabela 5 .

Os resultados dos testes apresentados na tabela 5 permitem afirmar que existe dimorfismo entre gêneros, com diferenças estatisticamente significantes, entre as seguintes medidas:

- Em xantodermas: AFAT (N-Me), AFAS (NENA'), AFPT (S-Go), AFPS (S-Ar') e AFA (Me$\mathrm{PP})$;

- Em leucodermas: AFPS (S-Ar'); AFPI/AFPT, AFPS/AFPT e IAF (AFP/ AFA).

Buscou-se, também, comparar as medidas analisadas, em xantodermas e leucodermas, de ambos os gêneros. Para isso, foram calculadas as diferenças entre médias de cada medida para cada gênero e feitas as diferenças, entre xantodermas e leucodermas. Depois foi aplicado o teste $t$ de Student, para diferença obtida (Tab. 6). 
Tabela 1 - Tamanho da amostra, média e desvio padrão de cada medida estudada do grupo xantodermas, gênero masculino.

\begin{tabular}{cccc}
\hline Medida & $\begin{array}{c}\text { Tamanho da } \\
\text { amostra }\end{array}$ & Média & Desvio padrão \\
\hline AFAT (N-Me) & 20 & 119,11 & 4,31 \\
AFAS (N-ENA') & 20 & 53,44 & 2,16 \\
AFAl (ENA'-Me) & 20 & 65,67 & 3,52 \\
AFAS/ AFAT & 20 & 44,92 & 1,62 \\
AFAl/ AFAT & 20 & 55,09 & 1,62 \\
AFPT (S-Go) & 20 & 80,01 & 5,08 \\
AFPS (S-Ar') & 20 & 33,68 & 2,49 \\
AFPI (Ar'-Go) & 20 & 46,33 & 4,57 \\
AFPS/ AFPT & 20 & 42,16 & 3,04 \\
AFPI/ AFPT & 20 & 57,85 & 3,04 \\
AFA (Me-PP) & 20 & 65,78 & 3,66 \\
AFP (Ar-PM) & 20 & 49,50 & 4,89 \\
IAF (AFP/AFA) & 20 & 75,32 & 6,98 \\
\hline
\end{tabular}

Tabela 3 - Tamanho da amostra, média e desvio padrão de cada medida estudada do grupo leucodermas, gênero masculino.

\begin{tabular}{cccc}
\hline Medida & $\begin{array}{c}\text { Tamanho da } \\
\text { amostra }\end{array}$ & Média & Desvio padrão \\
\hline AFAT (N-Me) & 39 & 113,33 & 6,18 \\
AFAS (N-ENA') & 39 & 50,46 & 2,85 \\
AFAI (ENA'-Me) & 39 & 62,87 & 4,71 \\
AFAS/ AFAT & 39 & 44,56 & 1,97 \\
AFAl/ AFAT & 39 & 55,44 & 1,97 \\
AFPT (S-Go) & 39 & 71,91 & 5,47 \\
AFPS (S-Ar') & 39 & 30,81 & 3,07 \\
AFPI (Ar'-Go) & 39 & 41,09 & 4,23 \\
AFPS/ AFPT & 39 & 42,88 & 3,36 \\
AFPI/ AFPT & 39 & 57,12 & 3,36 \\
AFA (Me-PP) & 39 & 62,84 & 4,45 \\
AFP (Ar-PM) & 39 & 43,84 & 4,30 \\
IAF (AFP/AFA) & 39 & 69,81 & 5,17 \\
\hline
\end{tabular}

Os resultados dos testes, apresentados na tabela 6 , permitem afirmar que existe diferença significante entre xantodermas e leucodermas com relação às seguintes medidas:

- No gênero masculino: AFAT (N-Me), AFAS
Tabela 2 - Tamanho da amostra, média e desvio padrão de cada medida estudada do grupo xantodermas, gênero feminino.

\begin{tabular}{cccc}
\hline Medida & $\begin{array}{c}\text { Tamanho da } \\
\text { amostra }\end{array}$ & Média & Desvio padrão \\
\hline AFAT (N-Me) & 20 & 113,88 & 6,21 \\
AFAS (N-ENA') & 20 & 51,07 & 3,60 \\
AFAI (ENA'-Me) & 20 & 62,81 & 5,59 \\
AFAS/ AFAT & 20 & 44,90 & 3,03 \\
AFAl/ AFAT & 20 & 55,11 & 3,03 \\
AFPT (S-Go) & 20 & 74,63 & 5,50 \\
AFPS (S-Ar') & 20 & 30,60 & 3,55 \\
AFPI (Ar'-Go) & 20 & 44,05 & 3,47 \\
AFPS/ AFPT & 20 & 40,95 & 3,04 \\
AFPI/ AFPT & 20 & 59,05 & 3,04 \\
AFA (Me-PP) & 20 & 62,87 & 5,31 \\
AFP (Ar-PM) & 20 & 46,83 & 3,53 \\
IAF (AFP/AFA) & 20 & 74,92 & 7,97 \\
\hline
\end{tabular}

Tabela 4 - Tamanho da amostra, média e desvio padrão de cada medida estudada do grupo leucodermas, gênero feminino.

\begin{tabular}{cccc}
\hline Medida & $\begin{array}{c}\text { Tamanho da } \\
\text { amostra }\end{array}$ & Média & Desvio padrão \\
\hline AFAT (N-Me) & 39 & 111,58 & 4,98 \\
AFAS (N-ENA') & 39 & 49,92 & 2,59 \\
AFAI (ENA'-Me) & 39 & 61,66 & 4,39 \\
AFAS/ AFAT & 39 & 44,78 & 2,21 \\
AFAI/ AFAT & 39 & 55,22 & 2,21 \\
AFPT (S-Go) & 39 & 70,29 & 4,78 \\
AFPS (S-Ar') & 39 & 28,83 & 2,99 \\
AFPI (Ar'-Go) & 39 & 41,46 & 3,22 \\
AFPS/ AFPT & 39 & 40,99 & 2,87 \\
AFPI/ AFPT & 39 & 59,01 & 2,87 \\
AFA (Me-PP) & 39 & 61,42 & 4,28 \\
AFP (Ar-PM) & 39 & 44,54 & 3,57 \\
IAF (AFP/AFA) & 39 & 72,73 & 6,13 \\
\hline
\end{tabular}

(N-ENA'), AFAI (ENA'-Me), AFPT(S-Go), AFPS (S-Ar'), AFPI (Ar'-Go), AFA (Me-PP), AFP (ArPM) e IAF (AFP/AFA);

- No gênero feminino: AFPT (S-Go), AFPS (SAr'), AFPI (Ar'-Go) e AFP (Ar-PM). 
Tabela 5 - Diferenças entre gêneros e teste $t$ da diferença, para cada medida estudada.

\begin{tabular}{ccccc}
\hline \multirow{2}{*}{ Medida } & \multicolumn{2}{c}{ Xantodermas } & \multicolumn{2}{c}{ Leucodermas } \\
\cline { 2 - 5 } & Diferença & Teste $\boldsymbol{t}$ & Diferença & Teste $\boldsymbol{t}$ \\
\hline AFAT (N-Me) & 5,23 & $3,09^{*}$ & 1,74 & 1,37 \\
AFAS (N-ENA') & 2,37 & $2,52^{*}$ & 0,54 & 0,87 \\
AFAI (ENA'-Me) & 2,86 & 1,94 & 1,21 & 1,17 \\
AFAS/ AFAT & 0,02 & 0,03 & $-0,22$ & $-0,47$ \\
AFAl/ AFAT & $-0,02$ & $-0,03$ & 0,22 & 0,47 \\
AFPT (S-Go) & 5,38 & $3,21^{*}$ & 1,62 & 1,39 \\
AFPS (S-Ar') & 3,09 & $3,18^{*}$ & 1,98 & $2,89^{*}$ \\
AFPI (Ar'-Go) & 2,28 & 1,77 & $-0,37$ & $-0,44$ \\
AFPS/ AFPT & 1,21 & 1,25 & 1,89 & $2,68^{*}$ \\
AFPI/ AFPT & $-1,21$ & $-1,25$ & $-1,89$ & $-2,68^{*}$ \\
AFA (Me-PP) & 2,91 & $2,02^{*}$ & 1,42 & 1,44 \\
AFP (Ar-PM) & 2,67 & 1,98 & $-0,70$ & $-0,78$ \\
IAF (AFP/AFA) & 0,40 & 0,17 & $-2,92$ & $-2,27^{*}$ \\
\hline * significante ao nível de $5 \%$ & & &
\end{tabular}

Tabela 7 - Diferenças entre gêneros e intervalo de confiança, para cada medida estudada do grupo xantoderma.

\begin{tabular}{cccc}
\hline \multirow{2}{*}{ Medida } & Diferença & \multicolumn{2}{c}{ Limites do intervalo } \\
\cline { 3 - 4 } & & Inferior & Superior \\
\hline AFAT (N-Me) & 5,23 & 1,92 & 8,54 \\
AFAS (N-ENA') & 2,37 & 0,53 & 4,21 \\
AFAI (ENA'-Me) & 2,86 & $-0,04$ & 5,76 \\
AFAS/ AFAT & 0,02 & $-1,49$ & 1,53 \\
AFAI/ AFAT & $-0,02$ & $-1,53$ & 1,49 \\
AFPT (S-Go) & 5,38 & 2,09 & 8,66 \\
AFPS (S-Ar') & 3,09 & 1,19 & 4,98 \\
AFPI (Ar'-Go) & 2,28 & $-0,24$ & 4,79 \\
AFPS/ AFPT & 1,21 & $-0,68$ & 3,09 \\
AFPI/ AFPT & $-1,21$ & $-3,09$ & 0,68 \\
AFA (Me-PP) & 2,91 & 0,08 & 5,74 \\
AFP (Ar-PM) & 2,67 & 0,03 & 5,31 \\
IAF (AFP/AFA) & 0,40 & $-4,24$ & 5,04 \\
\hline
\end{tabular}

Também foram calculados intervalos (média \pm desvio padrão) para as diferenças entre gêneros, dentro de cada grupo racial, e intervalos (média \pm desvio padrão) para as diferenças entre raças, dentro de cada gênero. Os resultados estão
Tabela 6 - Diferenças entre raças e teste $t$ da diferença, para cada medida estudada.

\begin{tabular}{ccccc}
\hline \multirow{2}{*}{ Medida } & \multicolumn{2}{c}{ Masculino } & \multicolumn{2}{c}{ Feminino } \\
\cline { 2 - 5 } & Diferença & Teste $\boldsymbol{t}$ & Diferença & Teste $\boldsymbol{t}$ \\
\hline AFAT (N-Me) & 5,78 & $3,73^{*}$ & 2,29 & 1,54 \\
AFAS (N-ENA') & 2,98 & $4,10^{*}$ & 1,14 & 1,40 \\
AFAI (ENA'-Me) & 2,80 & $2,34^{*}$ & 1,15 & 0,86 \\
AFAS/ AFAT & 0,36 & 0,69 & 0,11 & 0,16 \\
AFAI/ AFAT & $-0,36$ & $-0,69$ & $-0,11$ & $-0,16$ \\
AFPT (S-Go) & 8,10 & $5,51^{*}$ & 4,34 & $3,14^{*}$ \\
AFPS (S-Ar') & 2,87 & $3,61^{*}$ & 1,77 & $2,02^{*}$ \\
AFPI (Ar'-Go) & 5,24 & $4,38^{*}$ & 2,59 & $2,85^{*}$ \\
AFPS/ AFPT & $-0,73$ & $-0,82$ & $-0,04$ & $-0,05$ \\
AFPI/ AFPT & 0,73 & 0,82 & 0,04 & 0,05 \\
AFA (Me-PP) & 2,93 & $2,54^{*}$ & 1,44 & 1,13 \\
AFP (Ar-PM) & 5,65 & $4,56^{*}$ & 2,28 & $2,33^{*}$ \\
IAF (AFP/AFA) & 5,50 & $3,43^{*}$ & 2,19 & 1,17 \\
\hline * significante ao nível de $5 \%$ & & &
\end{tabular}

Tabela 8 - Diferenças entre gêneros e intervalo de confiança, para cada medida estudada do grupo leucoderma.

\begin{tabular}{cccc}
\hline \multirow{2}{*}{ Medida } & Diferença & \multicolumn{2}{c}{ Intervalo de confiança } \\
\cline { 3 - 4 } & & Inferior & Superior \\
\hline AFAT (N-Me) & 1,74 & $-0,75$ & 4,24 \\
AFAS (N-ENA') & 0,54 & $-0,67$ & 1,75 \\
AFAI (ENA'-Me) & 1,21 & $-0,81$ & 3,23 \\
AFAS/ AFAT & $-0,22$ & $-1,15$ & 0,71 \\
AFAI/ AFAT & 0,22 & $-0,71$ & 1,15 \\
AFPT (S-Go) & 1,62 & $-0,66$ & 3,90 \\
AFPS (S-Ar') & 1,98 & 0,64 & 3,33 \\
AFPI (Ar'-Go) & $-0,37$ & $-2,04$ & 1,30 \\
AFPS/ AFPT & 1,89 & 0,51 & 3,28 \\
AFPI/ AFPT & $-1,89$ & $-3,28$ & $-0,51$ \\
AFA (Me-PP) & 1,42 & $-0,52$ & 3,36 \\
AFP (Ar-PM) & $-0,70$ & $-2,45$ & 1,06 \\
IAF (AFP/AFA) & $-2,92$ & $-5,43$ & $-0,40$ \\
\hline
\end{tabular}

nas tabelas 7, 8, 9 e 10. Os intervalos calculados ajudam o clínico a estabelecer expectativas, uma vez que se espera que as diferenças que possam ocorrer entre gêneros ou entre raças devam estar dentro dos limites calculados. 
Tabela 9 - Diferenças entre raças e intervalo de confiança, para cada medida estudada do gênero masculino.

\begin{tabular}{cccc}
\hline \multirow{2}{*}{ Medida } & \multirow{2}{*}{ Diferença } & \multicolumn{2}{c}{ Intervalo de confiança } \\
\cline { 3 - 4 } & & Inferior & Superior \\
\hline AFAT (N-Me) & 5,78 & 2,74 & 8,81 \\
AFAS (N-ENA') & 2,98 & 1,55 & 4,40 \\
AFAI (ENA'-Me) & 2,80 & 0,45 & 5,14 \\
AFAS/ AFAT & 0,36 & $-0,65$ & 1,36 \\
AFAI/ AFAT & $-0,36$ & $-1,36$ & 0,65 \\
AFPT (S-Go) & 8,10 & 5,22 & 10,98 \\
AFPS (S-Ar') & 2,87 & 1,31 & 4,43 \\
AFPI (Ar'-Go) & 5,24 & 2,89 & 7,58 \\
AFPS/ AFPT & $-0,73$ & $-2,48$ & 1,02 \\
AFPI/ AFPT & 0,73 & $-1,02$ & 2,48 \\
AFA (Me-PP) & 2,93 & 0,67 & 5,20 \\
AFP (Ar-PM) & 5,65 & 3,22 & 8,08 \\
IAF (AFP/AFA) & 5,50 & 2,36 & 8,65 \\
\hline
\end{tabular}

\section{DISCUSSÃO}

\section{Alturas Faciais Anteriores}

\section{AFAT - Altura Facial Anterior Total (N-Me)}

A altura facial anterior total possui crescimento na infância ${ }^{29}$, na adolescência ${ }^{29} \mathrm{e}$ na fase adulta ${ }^{1,29}$. O aumento crânio-caudal (vertical) da face foi verificado até a quarta década de vida ${ }^{22}$; entretanto, na senilidade, ocorre uma tendência à diminuição ${ }^{29}$. Os indivíduos com sobremordida profunda ou com faces curtas possuem uma diminuição crânio-caudal (vertical) da face $8,20,27,56,58,59,83,91,92$ e aqueles com mordida aberta anterior ou com faces longas ${ }^{4,8,13,45,53,54,55,59,62,66,68,80,81,94}$ possuem a AFAT aumentada. Os ângulos FMA, SN.PM e a altura vertical total da face anterior possuem relação íntima, onde, a cada aumento do FMA ${ }^{20,38}$ e do SN.PM ${ }^{20,34}$, ocorre um aumento da AFAT.

Na presente pesquisa, no grupo xantoderma, os valores encontrados de $119,11 \mathrm{~mm}$, para o gênero masculino, e $113,88 \mathrm{~mm}$, para ofeminino, apresentaram diferença estatística significante ao nível de $5 \%$,
Tabela 10 - Diferenças entre raças e intervalo de confiança, para cada medida estudada do gênero feminino.

\begin{tabular}{cccc}
\hline \multirow{2}{*}{ Medida } & Diferença & \multicolumn{2}{c}{ Intervalo de confiança } \\
\cline { 3 - 4 } & & Inferior & Superior \\
\hline AFAT (N-Me) & 2,29 & $-0,63$ & 5,21 \\
AFAS (N-ENA') & 1,14 & $-0,45$ & 2,74 \\
AFAI (ENA'-Me) & 1,15 & $-1,45$ & 3,75 \\
AFAS/ AFAT & 0,11 & $-1,24$ & 1,47 \\
AFAI/ AFAT & $-0,11$ & $-1,47$ & 1,24 \\
AFPT (S-Go) & 4,34 & 1,63 & 7,05 \\
AFPS (S-Ar') & 1,77 & 0,05 & 3,49 \\
AFPI (Ar'-Go) & 2,59 & 0,81 & 4,37 \\
AFPS/ AFPT & $-0,04$ & $-1,62$ & 1,54 \\
AFPI/ AFPT & 0,04 & $-1,54$ & 1,62 \\
AFA (Me-PP) & 1,44 & $-1,06$ & 3,95 \\
AFP (Ar-PM) & 2,28 & 0,37 & 4,20 \\
IAF (AFP/AFA) & 2,19 & $-1,48$ & 5,85 \\
\hline
\end{tabular}

indicando dimorfismo entre gêneros e maior desenvolvimento crânio-caudal (vertical) da face anterior para o gênero masculino. No grupo leucoderma, os valores encontrados de $113,33 \mathrm{~mm}$, para o gênero masculino e $111,58 \mathrm{~mm}$, para o feminino, não apresentaram diferença estatística significante ao nível de $5 \%$, indicando ausência de dimorfismo entre gêneros, entretanto, observa-se maior desenvolvimento crânio-caudal (vertical) da face anterior para o gênero masculino (Tab. 1, 2, 3, 4).

Os valores detectados nos leucodermas por Weinberg e Kronman ${ }^{88}$ de 108,7mm; por Schu$\mathrm{dy}^{70}$ de 117,8mm; por Johnson ${ }^{38}$ de 109,42mm; por Jones e Meredith ${ }^{39}$ de $114 \mathrm{~mm}$; por Scheideman et al. ${ }^{67}$ de $126,4 \mathrm{~mm}$ para o gênero masculino e $119,8 \mathrm{~mm}$ para o feminino; por $\mathrm{Bishara}^{7}$ de $107 \mathrm{~mm}$ para o gênero feminino e 108 para o masculino; por Siriwat e Jarabak ${ }^{74}$ de 112,2mm; pelo padrão Bolton ${ }^{58}$ de $119,8 \mathrm{~mm}$; indicam uma variabilidade para a medida AFAT; entretanto os valores obtidos, neste estudo, posicionaram-se entre os mínimos e máximos, encontrados na literatura. Os valores observados por Ishii, Deguchi e 


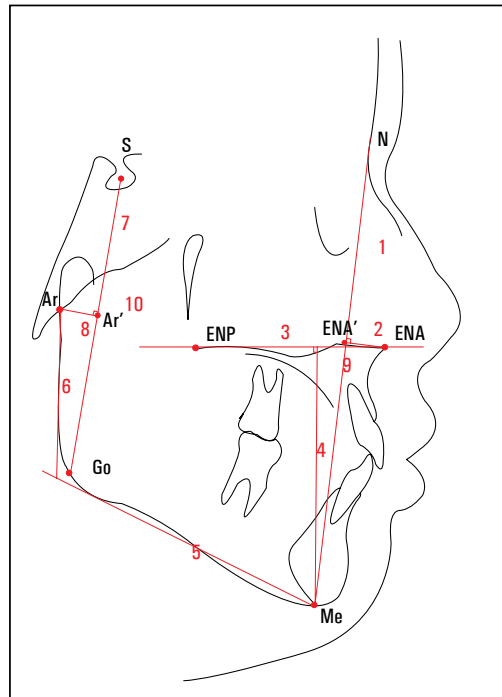

FIGURA 1 - Representação das linhas, planos e pontos construídos $\mathrm{Ar}^{\prime}$ e ENA'.

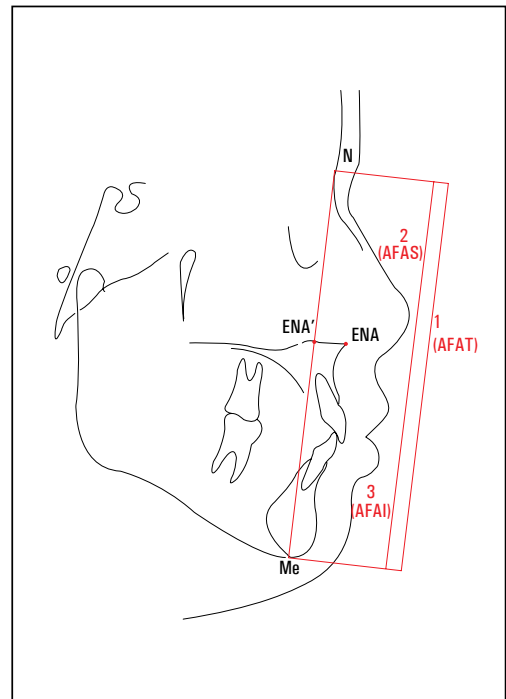

FIGURA 2 - Medidas empregadas de acordo com a Análise de Wylie e Johnson ${ }^{94}$.

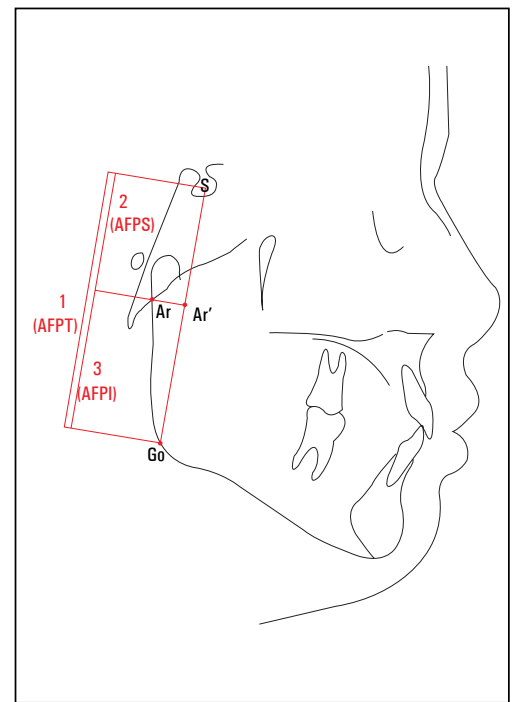

FIGURA 3 - Medidas empregadas de acordo com a Análise de Siriwat e Jarabak ${ }^{74}$
Hunt $^{35}$ de $122,8 \mathrm{~mm}$ demonstram ser maiores para os japoneses, em comparação ao encontrado nesta pesquisa.

Herzberg e Holic $^{31}$ verificaram uma semelhança entre as diferentes raças e observaram o valor de 115,2mm. No entanto, Ishii, Deguchi e Hunt $^{36}$ verificaram diferenças significantes entre xantodermas japoneses e leucodermas britânicos para ambos os gêneros, onde o grupo xantoderma apresentou valores maiores. No presente estudo, verificou-se diferença significante na comparação entre os grupos raciais, xantoderma e leucoderma, para o gênero masculino, sendo o valor maior para o grupo xantoderma. $\mathrm{O}$ gênero feminino não possuiu diferenças significantes entre os grupos raciais estudados, todavia o grupo xantoderma feminino apresentou valores maiores para AFAT.

\section{AFAS - Altura Facial Anterior Superior (N-ENA')}

A proximidade ao normal da face superior, representada pela AFAS, nos indivíduos com sobremordida profunda ou com faces cur$\operatorname{tas}^{20,45,58,59,62,70,92}$ e mordida aberta anterior ou com faces longas $4,20,45,54,59,62,68,80$, é verificada na literatu-

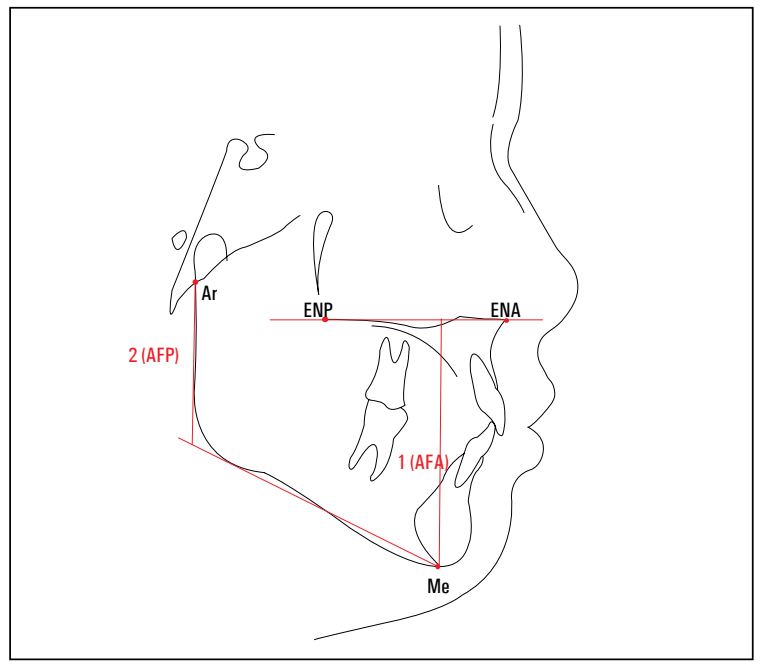

FIGURA 4 - Medidas empregadas de acordo com a Análise de Gebeck ${ }^{24}$, Merrifield ${ }^{51}$ e Horn ${ }^{32}$.

ra, onde as maiores variações ocorrem na face inferior. Porém, Nahoum ${ }^{53}$, observou a diminuição da altura facial superior nos indivíduos com mordida aberta anterior, devido possivelmente à inclinação do plano palatino; Trouten et al. ${ }^{83}$ verificaram, em adolescentes, e Toutountzakis e Haralabakis ${ }^{82}$, em adultos, maior desenvolvimento do complexo na- 
somaxilar em faces com mordida aberta anterior; Nanda ${ }^{56}$ verificou maior crescimento da AFAS, em indivíduos com sobremordida profunda; e Cheng et al. ${ }^{13}$ observaram aumento da AFAS em indivíduos com deficiência respiratória.

$\mathrm{Na}$ presente pesquisa, no grupo xantoderma, os valores encontrados de $53,44 \mathrm{~mm}$, para o gênero masculino e $51,07 \mathrm{~mm}$, para o feminino, apresentaram diferença estatística significante, ao nível de $5 \%$, indicando dimorfismo entre gêneros e maior desenvolvimento crânio-caudal (vertical) da face anterior superior para o masculino. No grupo leucoderma, os valores encontrados de 50,46mm, para o gênero masculino e $49,92 \mathrm{~mm}$, para o feminino, não apresentaram diferença estatística significante, ao nível de 5\%, denotando ausência de dimorfismo entre os gêneros e maior desenvolvimento crânio-caudal (vertical) da face anterior superior para o masculino (Tab. 1, 2, 3, 4).

$\mathrm{Na}$ literatura, verificam-se valores obtidos nos leucodermas por Herzberg e Holic ${ }^{31}$ de 50,3mm; por Wylie ${ }^{92}$ de $51,03 \mathrm{~mm}$; por $\mathrm{Schudy}^{70}$ de 51,3mm; por Weinberg e Kronman ${ }^{88}$ de 49,1mm; por Jones e Meredith ${ }^{39}$ de 52mm; por Domiti, Daruge e $\mathrm{Cruz}^{15}$ de $45,7 \mathrm{~mm}$ para o gênero masculino e $43,4 \mathrm{~mm}$ para o feminino, pelo padrão Bolton ${ }^{58}$ de $54,3 \mathrm{~mm}$; por Scheideman et al. ${ }^{67}$ de $56,0 \mathrm{~mm}$ para o gênero masculino e $53,7 \mathrm{~mm}$ para o feminino; por Bishara $^{7}$ de $47 \mathrm{~mm}$ para o gênero feminino e $48 \mathrm{~mm}$ para o masculino, mostraram uma semelhança entre os valores obtidos.

De acordo com os resultados obtidos por Jones, Meredith $^{39}$; Hunter ${ }^{33}$; Baume, Buschang, Weinstein ${ }^{3}$; Ursi et al..$^{85}$; Chang, Kinoshita e Kawamoto ${ }^{12}$, os indivíduos do gênero masculino apresentam um valor maior para a altura facial anterior superior, semelhante ao encontrado nesta pesquisa para os dois grupos raciais. Entretanto, Cannon ${ }^{11}$ observou maior desenvolvimento para a AFAS, no gênero feminino, enquanto Domiti, Daruge, Cruz $^{15}$ e Locks ${ }^{43}$ não observaram diferenças entre os gêneros.

Conforme Ishii, Deguchi e Hunt ${ }^{36}$, a altura fa- cial anterior superior foi significantemente maior para os indivíduos xantodermas japoneses, comparada aos indivíduos leucodermas britânicos. No presente estudo, verificou-se diferença significante na comparação entre os grupos raciais, xantoderma e leucoderma, para o gênero masculino; mas o feminino não possuiu diferenças significantes entre os grupos raciais estudados. Em ambos os gêneros, para as duas raças estudadas, observaram-se valores maiores para o grupo racial xantoderma.

\section{AFAl - Altura Facial Anterior Inferior (ENA'-Me)}

A altura facial anterior inferior sofre variações ambientais ${ }^{40}$, ocorrendo uma diminuição desta medida nos casos de sobremordida ou com síndrome da face curta $20,27,45,56,57,58,59,62,91,92$, e um aumento nos casos de mordida aberta anterior ou com síndrome da face longa ${ }^{4,20,45,53,55,56,57,59,62,68,80,82}$. A variação da AFAI também está relacionada à obstrução nasal ${ }^{13,60,81,90}$, apresentando aumento da AFAI em indivíduos com deficiência respiratória. $\mathrm{Na}$ face ântero-inferior, verifica-se a prevalência das variações verticais em se manifestar abaixo da espinha nasal anterior ${ }^{70}$, nas diferentes más oclusões e nos tipos faciais. As medidas FMA, SN.PM e AFAI possuem relação íntima, onde a cada aumento do FMA ${ }^{38}$ e do SN.PM ${ }^{34,81}$, ocorre um aumento da altura facial anterior inferior. De acordo com Kiliaridis et al. ${ }^{41}$, a altura facial ântero-inferior mostrou-se diminuída nos indivíduos com grande força mastigatória.

$\mathrm{Na}$ presente pesquisa, no grupo xantoderma, os valores encontrados de $65,67 \mathrm{~mm}$, para o gênero masculino e $62,81 \mathrm{~mm}$, para o feminino, não apresentaram diferença estatística significante, ao nível de 5\%, indicando ausência de dimorfismo entre os gêneros e maior desenvolvimento crâniocaudal (vertical) da face inferior para o masculino. No grupo leucoderma, os valores encontrados de $62,87 \mathrm{~mm}$, para o gênero masculino e $61,66 \mathrm{~mm}$, para o feminino, não apresentaram diferença estatística significante, ao nível de $5 \%$, indicando au- 
sência de dimorfismo entre os gêneros e, também, maior desenvolvimento crânio-caudal (vertical) da face ântero-inferior para o masculino (Tab. 1, 2, 3, 4).

Os valores encontrados por Herzberg e Holic ${ }^{31}$ de $65 \mathrm{~mm}$; por Wylie ${ }^{92}$ de 67,64mm; por Schu$\mathrm{dy}^{70}$ de 66,5mm; por Domiti, Darug e Cruz ${ }^{15}$ de $71,6 \mathrm{~mm}$ para o gênero masculino e $69 \mathrm{~mm}$ para o feminino, pelo padrão Bolton ${ }^{58}$ de $65,4 \mathrm{~mm}$; e por Scheideman et al. ${ }^{67}$ de $70,4 \mathrm{~mm}$ para o gênero masculino e $66,1 \mathrm{~mm}$ para o feminino apresentaram uma variação nos valores para AFAI, em que os valores encontrados, nesta pesquisa, foram menores.

Os indivíduos do gênero masculino apresentaram maior altura facial ântero-inferior $3,11,12,41,42,43$, semelhante ao encontrado nesta pesquisa para os dois grupos raciais. Entretanto Domiti, Daruge e $\mathrm{Cruz}^{15}$ não observaram diferenças entre os gêneros, e Ursi et al. ${ }^{85}$ verificaram que, até os 14 anos, existe uma semelhança entre os gêneros, e os valores são maiores para o masculino, após os 16 anos. Os indivíduos com AFAI aumentada possuem alturas dentárias maiores, comparadas aos com AFAI normal ${ }^{37}$.

De acordo com Ishii, Deguchi e Hunt ${ }^{36}$, a altura facial ântero-inferior foi significantemente maior para os indivíduos xantodermas japoneses, comparada à dos indivíduos leucodermas britânicos. No presente estudo, verificou-se diferença significante na comparação entre os grupos raciais, xantoderma e leucoderma, para o gênero masculino, todavia, o feminino não apresentou diferenças significantes entre os grupos raciais estudados. Os valores encontrados, nesta pesquisa, foram maiores para os xantodermas de ambos os gêneros.

\section{AFAS/AFAT - Proporção entre a Altura Facial Anterior Superior e Altura Facial Anterior Total (N-ENA'/ N-Me)}

A proporção entre altura facial anterior superior e altura facial anterior total apresenta normalidade, em faces equilibradas ${ }^{94}$; e variação, em fa- ces com desequilíbrio ${ }^{70}$. A proporção AFAS/AFAT mostrou ser constante, nas diferentes raças ${ }^{31}$, não apresentando dimorfismo entre gêneros ${ }^{94}$. Domiti, Daruge e Cruz ${ }^{15}$ observaram a manutenção das proporções faciais com o crescimento entre as idades de 6 e 15 anos.

$\mathrm{Na}$ presente pesquisa, no grupo xantoderma, os valores encontrados de 44,92\%, para o gênero masculino, e $44,90 \%$, para o feminino, não apresentaram diferença estatística significante ao nível de $5 \%$, indicando ausência de dimorfismo entre gêneros. No grupo leucoderma, os valores encontrados de $44,56 \%$, para o gênero masculino, e $44,78 \%$, para o feminino, não apresentaram diferença estatística significante ao nível de $5 \%$, indicando ausência de dimorfismo entre gêneros (Tab. $1,2,3,4)$.

Os valores encontrados em leucodermas por Herzberg e Holic ${ }^{31}$ de 43,51\%; por Wylie ${ }^{91}$ de $45,8 \%$; por Wylie e Johnson ${ }^{94}$ de $45 \%$; por Weinberg e Kronman ${ }^{88}$ de 45,14\%; por Jones e Meredith $^{39}$ de $44 \%$, para o gênero masculino e $44,5 \%$ para o feminino; por Bergersen ${ }^{5}$ de $44,41 \%$, para o gênero feminino e $43,60 \%$ para o masculino; por Bishara ${ }^{7}$ de $44 \%$, para o gênero feminino e $44 \%$ para o masculino, por Locks ${ }^{44}$ de $42 \%$; e por Chang, Kinoshita e Kawamoto ${ }^{12}$, em xantodermas chineses, de $45 \%$, evidenciam a semelhança com os resultados obtidos, na presente pesquisa, para ambos os grupos raciais.

No presente estudo, não se verificou diferença significante na comparação entre os grupos raciais, xantoderma e leucoderma, em ambos os gêneros, onde se observaram valores semelhantes para os dois grupos raciais e para ambos os gêneros, a denotar um equilíbrio nesta proporção.

\section{AFAl/AFAT - Proporção entre a Altura Facial Anterior Inferior e Altura Facial Anterior Total (ENA'-Me/ N-Me)}

A proporção entre altura facial anterior inferior e altura facial anterior total comporta-se de forma diferente, quando relacionada à face, apre- 
sentando um desequilíbrio, nas faces ruins ou pobres $^{94}$, e normalidade, em faces equilibradas ${ }^{94}$. Merow $^{52}$ observou menor crescimento da face inferior na proporção vertical. No entanto, a proporção AFAI/ AFAT mostrou-se constante nas diferentes raças ${ }^{31}$ e mantém-se com o crescimento ${ }^{15,44}$. A proporção entre a altura facial anterior inferior e a altura facial anterior total possui a tendência de aumentar com o aumento do ângulo SN.MP ${ }^{34}$.

$\mathrm{Na}$ presente pesquisa, no grupo xantoderma, os valores encontrados de $55,09 \%$, para o gênero masculino, e $55,11 \%$, para o feminino, não apresentaram diferença estatística significante, ao nível de $5 \%$, indicando ausência de dimorfismo entre os gêneros. No grupo leucoderma, os valores encontrados de $55,44 \%$, para o gênero masculino e $55,22 \%$, para o feminino, não apresentaram diferença estatística significante, ao nível de $5 \%$, indicando ausência de dimorfismo entre os gêneros (Tab. 1, 2, 3, 4).

Os valores encontrados em leucodermas por Herzberg e Holic ${ }^{31}$ de 56,49\%; por Wylie ${ }^{91}$ de $54,2 \%$; por Wylie e Johnson ${ }^{94}$ de $55 \%$; por Schu$\mathrm{dy}^{70}$ de 56,45\%; por Weinberg e Kronman ${ }^{88}$ de $54,86 \%$; por Jones e Meredith ${ }^{39}$ de 56\%; por Scheideman et al. ${ }^{67}$ de $55,5 \%$ para o gênero masculino e $55 \%$ para o feminino; por Locks ${ }^{44}$ de $58 \%$; e, em chineses, por Chang, Kinoshita e Kawamoto ${ }^{12}$ de $55 \%$, evidenciaram uma semelhança na proporção AFAI/AFAT com os valores obtidos nesta pesquisa para ambos os grupos raciais.

No presente estudo, não se verificou diferença significante na comparação entre os grupos raciais, xantoderma e leucoderma, em ambos os gêneros, evidenciando a constância na proporção facial AFAI/AFAT.

\section{Alturas Faciais Posteriores}

\section{AFPT - Altura Facial Posterior Total (S-Go)}

Os indivíduos com mordida aberta anterior ou com faces longas possuem a AFPT diminuí$\mathrm{da}^{8,54,59,66,68}$, devido à posição mais superior dos côndilos ${ }^{59,66}$, ao ramo mandibular curto ${ }^{66}$, à extrusão dos molares ${ }^{66}$ e inclinação dos planos oclusal e mandibular ${ }^{20,66}$; e os indivíduos com sobremordida profunda ou com faces curtas possuem a AFPT aumentada ${ }^{8,58}$. Entretanto, Fields et al. ${ }^{20}$ não encontraram diferenças na AFPT, em indivíduos com faces curtas, longas e normais; $\mathrm{Nanda}^{56}$ não verificou diferenças para AFPT, entre indivíduos com mordida aberta e sobremordida profunda; e Cheng et al. ${ }^{13}$ observaram ausência de aumento da AFPT, em indivíduos com deficiência respiratória.

$\mathrm{Na}$ presente pesquisa, no grupo xantoderma, os valores encontrados de $80,01 \mathrm{~mm}$, para o gênero masculino, e $74,63 \mathrm{~mm}$, para o feminino, apresentaram diferença estatística significante, ao nível de $5 \%$, indicando dimorfismo entre gêneros e maior desenvolvimento crânio-caudal (vertical) da face posterior para o masculino. No grupo leucoderma, os valores encontrados de $71,91 \mathrm{~mm}$, para o gênero masculino e $70,29 \mathrm{~mm}$, para o feminino, não apresentaram diferença estatística significante, ao nível de $5 \%$, indicando ausência de dimorfismo entre gêneros e maior desenvolvimento crânio-caudal (vertical) da face posterior para o masculino (Tab. $1,2,3,4)$.

Os valores encontrados por $\mathrm{Schudy}^{70}$ de $74,01 \mathrm{~mm}$, pelo padrão Bolton ${ }^{58}$ de $79 \mathrm{~mm}$; por Scheideman et al. ${ }^{67}$ de $92,9 \mathrm{~mm}$ para o gênero masculino e $84,2 \mathrm{~mm}$ para o feminino; por Bisha$\mathrm{ra}^{7}$ de $72 \mathrm{~mm}$ para o gênero feminino e $74 \mathrm{~mm}$ para o masculino; por Siriwat e Jarabak ${ }^{74}$ de $69,9 \mathrm{~mm}$ para o gênero feminino e $72,2 \mathrm{~mm}$ para o masculino, evidenciam a variabilidade da AFAT entre os diferentes autores e na presente pesquisa.

A dimensão crânio-caudal (vertical) posterior e a anterior da face demonstram maior crescimento no gênero masculino, tornando a face proporcionalmente maior que as faces femininas ${ }^{14}$. A altura facial posterior possui tendência a um menor crescimento que a altura facial anterior, nos indivíduos do gênero masculino, e os indivíduos do feminino apresentam um equilíbrio entre o crescimento crânio-caudal (vertical), posterior e anterior da 
face $^{14}$. Entretanto, Foley e Mamandras ${ }^{21}$ verificaram, no gênero feminino, que a altura facial posterior possuiu um suave aumento em relação à altura facial anterior, resultando em redução do ângulo do plano mandibular. Chang, Kinoshita e Kawamoto ${ }^{12}$ não encontraram diferenças entre os gêneros. De acordo com S. Bishara, Peterson e E. Bishara $^{9}$, a magnitude do aumento na altura facial posterior total no gênero masculino não foi significantemente diferente, nas idades entre 4,5 a 10 anos; 10 a 15 anos e 15 a 25,5 anos, todavia, no gênero feminino, verificou-se um aumento significante entre as idades de 4,5 a 10 anos.

$\mathrm{Na}$ presente pesquisa, verificou-se diferença significante na comparação entre os grupos raciais, xantoderma e leucoderma, para o gênero masculino e feminino, onde o grupo xantoderma apresentou valores maiores.

\section{AFPS - Altura Facial Posterior Superior (S-Ar')}

A síndrome da face longa pode estar associada ou não à mordida aberta anterior e possui a face superior posterior com valores próximos à normalidade $^{4,20}$. Os indivíduos com faces curtas ou sobremordida não possuem diferenças na $\mathrm{AFPS}^{20}$. De acordo com Linden ${ }^{42}$, a altura facial posterior superior apresenta maior crescimento do que a altura facial posterior inferior.

$\mathrm{Na}$ presente pesquisa, no grupo xantoderma, os valores encontrados de $33,68 \mathrm{~mm}$, para o gênero masculino, e $30,60 \mathrm{~mm}$, para o feminino, apresentaram diferença estatística significante, ao nível de 5\%, indicando dimorfismo entre gêneros e maior desenvolvimento crânio-caudal (vertical) da face posterior superior para o gênero masculino. No grupo leucoderma, os valores encontrados de $30,81 \mathrm{~mm}$, para o gênero masculino, e $28,83 \mathrm{~mm}$, para o feminino, apresentaram diferença estatística significante, ao nível de 5\%, indicando dimorfismo entre gêneros e maior desenvolvimento crâniocaudal (vertical) da face posterior superior para o masculino (Tab. 1, 2, 3, 4).

De acordo com Ishii, Deguchi e Hunt ${ }^{36}$, não foram verificadas diferenças entre xantodermas japoneses e leucodermas britânicos. No presente estudo, verificou-se diferença significante na comparação entre os grupos raciais, xantoderma e leucoderma, para o gênero masculino e feminino. Os indivíduos xantodermas apresentaram maiores valores, comparados aos leucodermas.

\section{AFPI - Altura Facial Posterior Inferior (Ar'-Go)}

$\mathrm{Na}$ face posterior, verifica-se a prevalência das variações verticais em se manifestarem abaixo da fossa glenóide ${ }^{70}$, nas diferentes más oclusões e tipos faciais. Os indivíduos, com mordida aberta anterior ou com faces longas, possuem uma tendência à menor altura facial posterior inferior ${ }^{8}$; entretanto, Subtelny e Sakuda ${ }^{80}$ não verificaram diferença estatística em indivíduos de oclusão normal e mordida aberta anterior, apesar de o grupo da mordida aberta ter apresentado valores menores; $\mathrm{e}$ os indivíduos com sobremordida profunda e faces curtas $^{8}$, maior AFPI. De acordo com Bishara, Peterson e Bishara ${ }^{9}$, a magnitude do aumento na altura facial posterior inferior, no gênero masculino, não foi significantemente diferente nas idades entre 4,5 a 10 anos; 10 a 15 anos e 15 a 25,5 anos.

$\mathrm{Na}$ presente pesquisa, no grupo xantoderma, os valores encontrados de $46,33 \mathrm{~mm}$, para o gênero masculino e $44,05 \mathrm{~mm}$, para o feminino, não apresentaram diferença estatística significante, ao nível de 5\%, indicando ausência de dimorfismo entre gêneros e maior desenvolvimento crâniocaudal (vertical) da face posterior inferior para o masculino. No grupo leucoderma, os valores encontrados de $41,09 \mathrm{~mm}$, para o gênero masculino e 41,46mm, para o feminino, não apresentaram diferença estatística significante, ao nível de 5\%, indicando ausência de dimorfismo entre gêneros (Tab. 1, 2, 3, 4).

Os valores encontrados, em leucodermas, por Schudy $^{70}$ de $45,8 \mathrm{~mm}$; por Bishara ${ }^{7}$ de $47 \mathrm{~mm}$ para o gênero feminino, e $48 \mathrm{~mm}$ para o masculino, para leucodermas; e por Ishii, Deguchi e Hunt ${ }^{35}$ de $47 \mathrm{~mm}$, em xantodermas japoneses, apresentam 
diferença entre os valores encontrados pela presente pesquisa.

No presente estudo, verificou-se diferença significante na comparação entre os grupos raciais, xantoderma e leucoderma, em ambos os gêneros, onde os indivíduos xantodermas apresentaram valores maiores.

\section{AFPS/AFPT - Proporção entre a Altura Facial Posterior Superior e Altura Facial Posterior To- tal (S-Ar'/ S-Go)}

$\mathrm{Na}$ presente pesquisa, no grupo xantoderma, os valores encontrados de $42,16 \%$, para o gênero masculino, e 40,95\%, para o feminino, não apresentaram diferença estatística significante ao nível de $5 \%$, indicando ausência de dimorfismo entre gêneros e maior proporção AFPS/AFPT para o masculino. No grupo leucoderma, os valores encontrados de $42,88 \%$, para o gênero masculino, e $40,99 \%$, para o feminino, apresentaram diferença estatística significante ao nível de $5 \%$, indicando dimorfismo entre gêneros e maior proporção AFPS/AFPT para o masculino (Tab. 1, 2, 3, 4).

No presente estudo, não se verificou diferença significante na comparação entre os grupos raciais, xantoderma e leucoderma, para os gêneros masculino e feminino.

AFPI/AFPT - Proporção entre a Altura Facial Posterior Inferior e Altura Facial Posterior Total (Ar'-Go/ S-Go)

De acordo com Bishara, Peterson e Bishara ${ }^{9}$, as mudanças na proporção entre AFPI/AFPT mostraram-se semelhantes para ambos os gêneros, sendo esta proporção menor nas idades entre 4,5 e 10 anos e maior nas idades entre 10 e 25,5 anos.

$\mathrm{Na}$ presente pesquisa, no grupo xantoderma, os valores encontrados de $57,85 \%$, para o gênero masculino, e $59,05 \%$, para o feminino, não apresentaram diferença estatística significante, ao nível de $5 \%$, indicando ausência de dimorfismo entre gêneros e maior proporção AFPI/AFPT para o feminino. No grupo leucoderma, os valores en- contrados de $57,12 \%$, para o gênero masculino, e $59,01 \%$, para o gênero feminino, apresentaram diferença estatística significante, ao nível de 5\%, indicando dimorfismo entre gêneros e maior proporção AFPI/AFPT para o feminino (Tab. 1, 2, 3, 4).

Os valores encontrados por Bishara ${ }^{7}$, em leucodermas, de $65 \%$ para o gênero feminino, e $65 \%$, para o masculino, diferem dos valores encontrados, nesta pesquisa.

No presente estudo, não se verificou diferença significante na comparação entre os grupos raciais, xantoderma e leucoderma, para o gênero masculino e feminino.

\section{Determinação do Índice da Altura Facial (IAF)}

O índice da altura facial, descrito por Horn ${ }^{32}$, é constituido pela razão entre a altura facial posterior (AFP) e altura facial anterior (AFA). Os indivíduos, com sobremordida profunda ou com a dimensão crânio-caudal (vertical) diminu$i_{\text {ida }}^{6,70}$, apresentam um maior índice; e os indivíduos, com tendência à mordida aberta anterior ou com a dimensão crânio-caudal (vertical) anterior aumentada ${ }^{6,70}$, um menor índice.

\section{AFA - Altura Facial Anterior (Me-PP)}

Nesta pesquisa, o grupo xantoderma apresentou valores de $65,78 \mathrm{~mm}$, para o gênero masculino, e $62,87 \mathrm{~mm}$, para o feminino, e teve diferença estatística significante ao nível de $5 \%$, indicando dimorfismo entre gêneros e maior desenvolvimento crânio-caudal (vertical) da face anterior inferior para o masculino. No grupo leucoderma, os valores encontrados de $62,84 \mathrm{~mm}$, para o gênero masculino, e $61,42 \mathrm{~mm}$, para o feminino, não apresentaram diferença estatística significante, ao nível de $5 \%$, indicando ausência de dimorfismo entre gêneros e maior desenvolvimento crânio-caudal (vertical) da face anterior inferior, para o masculino (Tab. 1, 2, 3, 4).

Os valores observados por Merrifield ${ }^{50}$ de $70 \mathrm{~mm}$, para a AFA, sendo $65 \mathrm{~mm}$ para o feminino, e $75 \mathrm{~mm}$ para o masculino; por Horn ${ }^{32}$ de $60 \mathrm{~mm}$, 
com uma variação de 39 a $80 \mathrm{~mm}$; por Merrifield ${ }^{51}$ de 67,90mm; e por Schott e Capelli Júnior ${ }^{69}$ de $63,8 \mathrm{~mm}$, para o feminino, e $72,47 \mathrm{~mm}$, para o masculino, em leucodermas brasileiros, mostraram diferença com os encontrados nesta pesquisa.

Verificou-se, neste estudo, diferença significante na comparação entre os grupos raciais, xantoderma e leucoderma, para o gênero masculino, e ausência de diferenças significantes entre os grupos raciais estudados para o feminino. Em ambos os gêneros, os indivíduos xantodermas possuíram valores maiores em comparação aos leucodermas.

\section{AFP - Altura Facial Posterior (Ar-PM)}

$\mathrm{Na}$ presente pesquisa, os valores encontrados em xantoderma japoneses, de $49,50 \mathrm{~mm}$ para o gênero masculino e $46,83 \mathrm{~mm}$ para o feminino, não apresentaram diferença estatística significante ao nível de 5\%, indicando ausência de dimorfismo entre gêneros e maior desenvolvimento crâniocaudal (vertical) da face posterior inferior para o masculino. No grupo leucoderma, os valores encontrados de $43,84 \mathrm{~mm}$, para o gênero masculino e $44,54 \mathrm{~mm}$, para o feminino, não apresentaram diferença estatística significante, ao nível de 5\%, indicando ausência de dimorfismo entre gêneros e maior desenvolvimento crânio-caudal (vertical) da face posterior inferior para o feminino (Tab. 1, 2, 3, 4).

$\mathrm{Na}$ literatura, encontraram-se os seguintes valores para a altura facial posterior: $41 \mathrm{~mm}^{32}$, por $\operatorname{Horn}^{32}$, com uma variação de 30 a $60 \mathrm{~mm}$; $50,70 \mathrm{~mm}$, por Merrifield ${ }^{51}$; $43 \mathrm{~mm}$ para o gênero feminino e $51,27 \mathrm{~mm}$ para o masculino por Schott e Capelli Júnior ${ }^{69}$, em leucodermas brasileiros.

No presente estudo, verificou-se diferença significante na comparação entre os grupos raciais, xantoderma e leucoderma, para o gênero masculino e feminino.

\section{IAF - Índice da Altura Facial (AFP/AFA) \\ O deslocamento da mandíbula, no sentido} horário ou anti-horário, pode ser o resultado da desarmonia entre o crescimento crânio-caudal (vertical) e ântero-posterior das estruturas craniofaciais, podendo promover implicações no tratamento ortodôntico. De acordo com Horn ${ }^{32}$, o índice de altura facial (FHI) permite o monitoramento da dimensão crânio-caudal (vertical) do paciente, durante o tratamento ortodôntico, por ser o controle da dimensão crânio-caudal (vertical) muito importante nos casos de FMA elevado e FHI diminuídos.

$\mathrm{Na}$ presente pesquisa, no grupo xantoderma, os valores encontrados, de $75,32 \%$ para o gênero masculino e $74,92 \%$ para o feminino, não apresentaram diferença estatística significante, ao nível de $5 \%$, indicando ausência de dimorfismo entre gêneros e maior índice da altura facial para o masculino. No grupo leucoderma, os valores encontrados, de $69,81 \%$ para o gênero masculino e $72,73 \%$ para o feminino, apresentaram diferença estatisticamente significante, ao nível de $5 \%$, indicando dimorfismo entre gêneros e maior desenvolvimento crânio-caudal (vertical) da face anterior inferior para o feminino (Tab. 1, 2, 3, 4).

$\mathrm{Na}$ literatura, verificou-se os seguintes valores para leucodermas: por $\mathrm{Schudy}^{70}$ de $68 \%$; por Biggerstaff et al. ${ }^{11}$ de $66 \%$ para o gênero masculino e $68 \%$ para o feminino; por Horn ${ }^{32}$ de $70 \%$; por Schott, Capelli Jr. ${ }^{69}$, em leucodermas brasileiros, de $67 \%$ para o gênero feminino e $70 \%$ para o masculino.

No presente estudo, verificou-se diferença significante na comparação entre os grupos raciais, xantoderma e leucoderma, para o gênero masculino; entretanto, o feminino não possuiu diferenças significantes, entre os grupos raciais estudados.

\section{CONSIDERAÇÕES CLÍNICAS COM RELAÇÃO ÀS ALTURAS FACIAIS E O CRESCIMENTO E DESENVOLVIMENTO DA FACE}

A desarmonia entre o crescimento crânio-caudal (vertical) e o ântero-posterior das estruturas craniofaciais pode promover a rotação mandibular, no sentido horário ou anti-horário, promo- 
vendo implicações no tratamento ortodôntico ${ }^{57,71}$. De acordo com Schudy ${ }^{71}$, 27\% do crescimento crânio-caudal (vertical) total da face ocorrem na mandíbula; e $73 \%$ na maxila. Dos $73 \%$ do crescimento crânio-caudal (vertical) da maxila, $56,5 \%$ ocorrem no corpo da maxila; e 43,5\% nos processos alveolares.

O desenvolvimento da sobremordida profunda, da mordida aberta anterior ou da sobremordida normal depende do relacionamento entre o crescimento dos côndilos mandibulares, do crescimento crânio-caudal (vertical) do corpo da maxila, do desenvolvimento crânio-caudal (vertical) dos processos alveolares posteriores da maxila e mandíbula e do desenvolvimento crânio-caudal (vertical) dos processos alveolares anteriores, da maxila e mandíbula ${ }^{72}$. A musculatura pode influenciar no desenvolvimento total da dimensão crânio-caudal (vertical) ${ }^{10,91}$, causando uma sobremordida.

As mudanças decorrentes do tratamento ortodôntico podem ser desejáveis ou indesejáveis $^{23,48,61,75,85}$, com as seguintes possibilidades: a) aumento harmonioso na altura facial anterior e posterior, promovendo uma constância na proporção entre as alturas faciais, superior e inferior, bem como um equilíbrio na proporção entre a altura facial posterior inferior e a altura facial ântero-inferior; b) aumento na altura facial anterior, sem o aumento proporcional da altura facial posterior, associado a um movimento inferior do mento e rotação horária da mandíbula, promovendo aumento das alturas faciais anteriores, superior, inferior, total e mudanças na proporção entre as alturas faciais inferiores, anterior e posterior; e c) aumento na altura facial posterior, sem aumento proporcional na altura facial anterior, proporcionando a rotação anti-horária da mandíbula, ocorrendo mudanças na proporção entre as alturas faciais inferiores, posterior e anterior.

A má oclusão de Classe II possui características próprias ${ }^{30,63,64}$, como o aumento da $\mathrm{AFAI}^{30}$, e seu tratamento deve possibilitar o aumento da AFP ${ }^{23,}$ 24,25,26,32,51,61,86 e minimizar o da $\mathrm{AFA}^{23,24,26,32,51,61,86}$, principalmente em pacientes em fase de crescimento, com FMA elevado e índice da altura facial diminuído, a fim de proporcionar um equilíbrio das estruturas esqueléticas, dentárias e tegumentares. O sucesso do tratamento da má oclusão de Classe II, primeira divisão, está relacionado ao controle da dimensão crânio-caudal (vertical), onde os tratamentos com sucesso ${ }^{24,26,32,51,86}$ apresentam pouco ou nenhum aumento da altura facial anterior (AFA), aumento da altura facial posterior (AFP) e rotação anti-horária da mandíbula; e os tratamentos com insucesso apresentam aumento da $\mathrm{AFA}^{24,26,32,51,86}$ e rotação horária da mandíbula. Os cuidados, no controle da dimensão crânio-caudal (vertical), em pacientes de Classe II, $1^{\text {a }}$ divisão, com padrão de crescimento equilibrado, são menores, pois são pequenas as alterações nas alturas faciais e no índice facial, decorrentes do tratamento ortodôntico ${ }^{28,46}$.

A partir dos dados referentes aos valores normativos de xantodermas e leucodermas, no sentido crânio-caudal (vertical) da face, encontrados nesta pesquisa, o clínico poderá utilizá-los como referência no diagnóstico e no plano de tratamento ortodôntico, considerando as diferenças raciais e as entre os gêneros.

\section{CONCLUSÕES}

Em função dos resultados obtidos e da metodologia utilizada, baseados em duas amostras de jovens, descendentes de xantodermas e leucodermas, com oclusão normal, julga-se lícito concluir o seguinte:

- Existência de valores médios de normalidade específicos para as grandezas cefalométricas esqueléticas, no sentido crânio-caudal (vertical) da face, para os jovens brasileiros xantodermas, descendentes de japoneses, com oclusão normal e idade média de 15,71 anos;

- Existência de valores médios de normalidade para as grandezas cefalométricas esqueléticas, no sentido crânio-caudal (vertical) da face, para os jovens brasileiros leucodermas, descendentes de 
grupos de origem mediterrânea (espanhóis, portugueses e italianos), com oclusão normal e idade média de 13,5 anos;

- Existência de dimorfismo entre gêneros, com diferenças estatisticamente significantes, entre as seguintes medidas:

- Em xantodermas: AFAT, altura facial anterior total (N-Me); AFAS altura facial anterior superior (N-ENA'); AFPT, altura facial posterior total (S-Go); AFPS, altura facial posterior superior (SAr'); e AFA, altura facial anterior (Me-PP);

- Em leucodermas: AFPS, altura facial posterior superior (S-Ar'); AFPI/AFPT, proporção entre altura facial posterior inferior e altura facial posterior total; AFPS/AFPT, proporção entre altura facial posterior superior e altura facial posterior total; e IAF, índice da altura facial (AFP/ AFA); e

- Diferença significante entre os grupos raciais, xantodermas e leucodermas, com relação às seguintes medidas:

- No gênero masculino: AFAT, altura facial anterior total (N-Me); AFAS, altura facial anterior superior (N-ENA'); AFAI, altura facial anterior inferior (ENA'-Me); AFPT, altura facial posterior total (S-Go); AFPS, altura facial posterior superior (S-Ar'); AFPI, altura facial posterior inferior (Ar' - Go); AFA, altura facial anterior (Me-PP); AFP, altura facial posterior (Ar - PM); e IAF, índice da altura facial (AFP/ AFA);

- No gênero feminino: AFPT, altura facial posterior total (S - Go); AFPS, altura facial posterior superior ( $\mathrm{S}$ - Ar'); AFPI, altura facial posterior inferior (Ar'-Go); e AFP, altura facial posterior (Ar - PM).

Enviado em: Abril de 2003 Revisado e aceito: Junho de 2003

Cephalometric comparative analysis of facial heights, anterior and posterior, in brazilian youths, descendents of japanese and caucasian, with normal occlusion

\begin{abstract}
Aim: this study was meant to verify the cephalometrics of the skeletal structures of the face in the vertical direction in order to obtain the mean values of normality for brazilian youths, japanese and caucasian descendents, with normal occlusion; to verify the presence or absence of dimorphism between the genders; and to investigate the differences or similarities between those racial groups. Methods: cephalometric evaluation. Results: after the determination of the average and standard deviation for each cephalometric measure under study, in both genders and racial groups, the results of the research evidenced, in some cephalometric measures, the presence of dimorphism between the genders in both racial groups and difference between the racial groups. Conclusions: it was verified the need to use a specific cephalometric standard, in the vertical direction, for brazilians, japanese and caucasian descendents.
\end{abstract}

Key words: Cephalometric standards. Normal occlusion. Vertical dimension.

\section{REFERÊNCIAS}

1. AHN, J.; SCHNEIDER, B. Cephalometric appraisal of posttreatment vertical changes in adult orthodontic patients. Am J Orthod Dentofacial Orthop, St. Louis, v. 118, no. 4, p. 378-384, Oct. 2000.
2. ANGLE, E. H. Classification of malocclusion. Dental Cosmos, Philadelphia, v. 41, no. 2, p. 248-264, Feb. 1899.

3. BAUME, R. M.; BUSCHANG, P. H.; WEINSTEIN, S. Stature, head height, and growth of the vertical face. Am J Orthod, St. Louis, v. 83, no. 6, p. 477-484, June 1983.

4. BELL, W. H.; CREEKMORE, T. D.; ALEXANDER, R. G. Surgical correction of the long face syndrome. Am J Orthod, St. Louis, v.71, no.1, p. 40-67, Jan. 1977. 
5. BERGERSEN, E. O. The directions of growth from infancy to adulthood. Angle Orthod, Appleton, v. 36, no. 1, p. 18-43, Jan. 1966.

6. BIGGERSTAFF, R. H. et al. A vertical cephalometric analysis of the human craniofacial complex. Am J Orthod, St. Louis, v. 72 no. 4, p. 397-405, Oct. 1977.

7. BISHARA, S. E. Longitudinal cephalometric standards from 5 years of age to adulthood. Am J Orthod, St. Louis, v.79, no.1, p. 35-44, Jan. 1981.

8. BISHARA, S. E.; JAKOBSEN, J. R. Longitudinal changes in three normal facial types. Am J Orthod, St. Louis, v. 88 , no. 6 , p. 466-502, Dec. 1985.

9. BISHARA, S. E.; PETERSON, L. C.; BISHARA, E. C. Changes in facial dimensions and relationships between the ages of 5 and 25 years. Am J Orthod, St. Louis, v. 85, no. 3, p. 238-252, Mar. 1984

10. BJÖRK, A. Prediction of mandibular growth rotation. Am J Orthod, St. Louis, v. 55, no. 6, p. 585-599, June 1969.

11. CANNON, J. Craniofacial height and depth increments in normal children. Angle Orthod, Appleton, v. 40, no. 3, p. 202-218, July 1970

12. CHANG, H. P.; KINOSHITA, Z.; KAWAMOTO, T. A study of the growth changes in facial configuration. Eur J Orthod, Oxford, v.15, no. 6, p. 493-501, Dec. 1993.

13. CHENG, M. C. et al. Developmental effects of impaired breathing in the face of the growing child. Angle Orthod, Appleton, v. 58, no. 4, p. 309-320, Oct. 1988.

14. COBEN, S. E. The integration of facial skeletal variants: a serial cephalometric roentgenographic analysis of craniofacial form and growth. Am J Orthod, St. Louis, v. 41, no. 6, p. 407-434, June 1955.

15. DOMITI, S. S.; DARUGE, E.; CRUZ, V. F. Variability of the nasion-subnasal, subnasal-gnathion, and bizygomatic distances of individuals of $6,7,11$, and 15 years of age and their importance in the determination of the vertical dimension. Aust Dent $\mathbf{J}$, Sydney, v. 21, no. 3, p. 269-271, June 1976.

16. DOWNS, W. B. Variations in facial relationships: their significance in treatment and prognosis. Am J Orthod, St. Louis, v. 34 , no. 10 , p. $812-840$, Oct. 1948

17. DOWNS, W. B. The role cephalometrics in orthodontic case analysis and diagnosis Am J Orthod, St. Louis, v. 38, no. 3, p.162-182, Mar. 1952

18. DOWNS, W. B. Analysis of the dentofacial profile. Angle Orthod, Appleton, v. 26, no. 4, p.191-212, Oct. 1956.

19. DOWNS, W. B. Quantitative and qualitative variations in facial growth. In: KRAUS, B.S.; RIEDEL, R. A. Vistas in Orthodontics. Philadelphia: Lea \& Febiger, 1962. p.188-195.

20. FIELDS, H. W. et al. Facial pattern differences in long-faced children and adults. Am J Orthod, St. Louis, v. 85, no. 3, p. 217-223, Mar. 1984.

21. FOLEY, T. F.; MAMANDRAS, A. H. Facial growth in females 14 to 20 years of age. Am J Orthod, St. Louis, v.101, no. 3, p. 248-254, Mar. 1992.

22. FORSBERG, C. M.; ELIASSON, S.; WESTERGREN, H. Face height and tooth eruption in adults: a 20 year follow-up investigation. Eur J Orthod, Oxford, v. 13, no. 4, p. 249-54, Aug. 1991.

23. FOTIS, V. et al. Vertical control as an important ingredient in the treatment of severe sagittal discrepancies. Am J Orthod, St. Louis, v. 86, no. 3, p. 224-231, Sept. 1984.

24. GEBECK, T. R. Analysis concepts and values. Part I. J Charles H Tweed Int Found, Menlo Park, v.17, p.19-48, Apr. 1989.

25. GEBECK, T. R.; MERRIFIELD, L. Orthodontic diagnosis and treatment analysis: concepts and values. Part I. Am J Orthod Dentofacial Orthop, St. Louis, v.107, no. 4, p. 434-443, Apr. 1995.

26. GEBECK, T. R.; MERRIFIELD, L. Orthodontic diagnosis and treatment analysis: concepts and values: Part II. Am J Orthod Dentofacial Orthop, St. Louis, v.107, no. 5, p. 541-547, May 1995.

27. GONDIM, P. P. C. et al. Estudo cefalométrico longitudinal do desenvolvimento da sobremordida em jovens leucodermas brasileiros dos 6 aos 18 anos. R Dental Press Ortodon Ortop Facial, Maringá, v. 6, n. 4, p. 7-17, jul./ago. 2001.

28. HAYASAKI, S.M. Estudo Cefalométrico das alterações das alturas faciais anterior e posterior em pacientes nipo-brasileiros com más oclusões de Classe I e Classe II, 1a divisão de Angle, tratados ortodonticamente. 2002. 196 f. Dissertação (Mestrado)-Faculdade de Odontologia de Bauru, Universidade de São Paulo, Bauru, 2002.
29. HELLMAN, M. A preliminary study in development as it affects the human face. Dental Cosmos, Philadelphia, v. 69, no. 3, p. 250-269, Mar. 1927

30. HENRIQUES, J. F. C. et. al. Estudo cefalométrico longitudinal das características da má-oclusão de Classe II, 1a divisão sem tratamento, em jovens brasileiros, leucodermas, por um período médio de 3 anos e 4 meses. R Dental Press Ortodon Ortop Facial, Maringá, v. 3, n. 3, p. 52-66, maio./ jun. 1998.

31. HERZBERG, F.; HOLIC, R. An anthropologic study of face height. Am J Orthod Oral Surg, St. Louis, v. 29, no. 2, p. 90-100, Feb. 1943.

32. HORN, A. J. Facial height index. Am J Orthod Dentofacial Orthop, St. Louis, v. 102, no. 2, p.180-186. Aug. 1992.

33. HUNTER, W. S. The vertical dimensions of the face and skeletodental retrognathism. Am J Orthod, St. Louis, v. 53, no. 8, p. 586-595, Aug. 1967.

34. ISAACSON, J. R. et al. Extreme variation in vertical facial growth and associated variation in skeletal and dental relations. Angle Orthod, Appleton, v. 41, no. 3, p. 219-229, July 1971.

35. ISHII, N.; DEGUCHI, T.; HUNT, N.P. Craniofacial morphology of Japanese girls with Class II division 1 malocclusion. J Orthod, London, v. 28, no. 3, p. 211-215, Sept. 2001.

36. ISHII, N.; DEGUCHI, T.; HUNT, N. P. Morphological differences in the craniofacial structure between Japanese and Caucasian girls with Class II division 1 malocclusions. Eur J Orthod, Oxford, v. 24, no. 3, p. 61-67, Sept. 2002.

37. JANSON, G. R. P.; METAXAS, A.; WOODSIDE, D. G. Variation in maxillary and mandibular molar and incisor vertical dimension in 12-year-old subjects with excess, normal, and short lower face height. Am J Orthod Dentofacial Orthop, St. Louis, v. 106, no. 4, p. 409-418, Oct. 1994.

38. JOHNSON, E. L. The Frankfort mandibular plane angle and the facial pattern. Am J Orthod, St. Louis, v. 36, no. 7, p. 516-533, July 1950.

39. JONES, B. H.; MEREDITH, H. V. Vertical change in osseous and odontic portions of human face height between the ages of 5 and 15 years. Am J Orthod, St. Louis, v. 52, no. 12, p. 902-921, Dec. 1966

40. KERR, W. J. S.; FORD, I. The variability of some craniofacial dimensions. Angle Orthod, Appleton, v. 61, no. 3, p. 205-210, Fall 1991.

41. KILIARIDIS, S. et al. The relationship between maximal bite force, bite force endurance, and facial morphology during growth. Acta Odontol Scand, Oslo Scandinavian, v. 51, no. 5, p. 323-331, Oct. 1993.

42. LINDEN, V. D. The facial skeleton in children from 7 to 11 years of age: a longitudinal roentgenocephalometric study. Am J Orthod, St. Louis, v. 46, no. 6, p. 470-472, June 1959.

43. LOCKS, A. Análise das proporções verticais anteriores da face de indivíduos brasileiros, portadores de oclusão excelente e perfil agradável. 1981. 69 f. Dissertação (Mestrado)-Universidade Federal do Rio de Janeiro, Rio de Janeiro, 1981.

44. LOCKS, A. Estudo cefalométrico das alturas faciais anterior e posterior, em crianças brasileiras, portadoras de má-oclusão Classe I de Angle, na fase de dentadura mista. 1996. 127 f. Tese (Doutorado)-Faculdade de Odontologia de Araraquara, Universidade Estadual Paulista "Júlio de Mesquita Filho", Araraquara, 1996.

45. LOUFTY, M. S. Cephalometric evaluation of deep overbite and anterior open bite in Kuwait school children. Trans Eur Orthod Soc, London, p. 281-285, 1973.

46. MACHADO, D.T. Estudo cefalométrico das alterações das alturas faciais anterior e posterior em pacientes leucodermas, com má oclusão de Classe II, 1a divisão de Angle, tratados com e sem extração de quatro primeiros pré-molares. 2002. 170 f. Dissertação (Mestrado)-Faculdade de Odontologia de Bauru, Universidade de São Paulo, Bauru, 2002.

47. MARTINS, D. R. et al. Atlas de crescimento craniofacial. São Paulo: Ed. Santos, 1998.

48. McNAMARA JR., J. A. An experimental study of increased vertical dimension in the growing face. Am J Orthod, St. Louis, v. 71, no. 4, p. 382-395, Apr. 1977

49. MEREDITH, H. V.; KNOTT, V. B.; HIXON, E. H. Relation of the nasal and subnasal components of facial height in childhood. Am J Orthod, St. Louis, v. 43, no. 5, p. 285-294, Apr. 1958. 
50. GEBECK, T. R.: MERRIFIELD, L. L. Orthodontic diagnosis and treatment analysis: concepts and values: Part II. Am J Orthod Dentofacial Orthop, St. Louis, v.107, no. 5, p. 541-547, May 1995.

51. MERRIFIELD, L. L. Analysis-concepts and values. Part II. J Charles H Tweed Int Found, Menlo Park, v. 17, p. 49-64, Apr. 1989.

52. MEROW, W. W. A cephalometric statistical appraisal of dentofacial growth. Angle Orthod, Appleton, v.32, no. 4, p. 205-213, Oct. 1962.

53. NAHOUM, H. I. Vertical proportions and the palatal plane in anterior open-bite. Am J Orthod, St. Louis, v. 59, no. 3, p. 273-282, Mar. 1971

54. NAHOUM, H. I.; HOROWITZ, S. L.; BENEDICTO, E. A. Varieties of anterior open-bite. Am J Orthod, St. Louis, v. 61, no. 5, p. 486-492, May 1972

55. NAHOUM, H. I. Vertical proportions: a guide for prognosis and treatment in anterior open-bite. Am J Orthod, St. Louis, v. 72, no. 2, p. 128-146, Aug. 1977.

56. NANDA, S. K. Patterns of vertical growth in the face. Am J Orthod, St. Louis, v. 93, no. 2, p.103-116, Feb. 1988.

57. NIELSEN, I. L. Vertical malocclusions: etiology, development, diagnosis and some aspects of treatment. Angle Orthod, Appleton, v. 61, no. 4, p. 247-260, Winter 1991.

58. OPDEBEECK, H.; BELL, W. H. The short face syndrome. Am J Orthod, St. Louis, v. 73, no. 5, p. 499-511, May 1978.

59. OPDEBEECK, H. et al. Comparative study between the SFS and LFS rotation as a possible morphogenic mechanism. Am J Orthod, St. Louis, v. 74, no. 5, p. 509-521, Nov. 1978

60. PRINCIPATO, J. J. Upper airway obstruction and craniofacial morphology. Otolaryngol Head Neck Surg, Rochester, v.104, no. 6, p. 881-890, June 1991.

61. RADZIMINSKI, G. The control of horizontal planes in Class II treatment. J Charles H Tweed Int Found, Menlo Park, v.15, p. 125-140, Apr. 1987

62. RICHARDSON, A. Skeletal factors in anterior open-bite and deep overbite. Am J Orthod, St. Louis, v. 56, no. 2, p.114-127, Aug. 1969

63. ROTHSTEIN, T.; YOON-TARLIE, C. Dental and facial characteristics and growth of males and females with Class II, division 1 malocclusion between the ages of 10 and 14 (revisited) - Part I: characteristics of size, form, and position. Am J Orthod Dentofacial Orthop, St. Louis, v.117, no. 3, p. 320-332, Mar. 2000.

64. ROTHSTEIN, T.; YOON-TARLIE, C. Dental and facial characteristics and growth of females and males with Class II, division 1 malocclusion between the ages of 10 and 14 (revisited) - Part II: anteroposterior and vertical circumpubertal growth. Am J Orthod Dentofacial Orthop, St. Louis, v.120, no. 5, p. 542-555, Nov. 2001

65. SALZMANN, J. A. The research workshop on cephalometrics. Am J Orthod, St. Louis, v. 46, no. 11, p. 834-847, Nov. 1960.

66. SASSOUNI, V.; NANDA, S. Analysis of dentofacial vertical proportions. Am J Orthod, St. Louis, v. 50, no. 11, p. 801-823, Nov. 1964.

67. SCHEIDEMAN, G. B. et al. Cephalometric analysis of dentofacial normals. Am J Orthod, St. Louis, v. 78, no. 4, p. 404-420, Oct. 1980

68. SCHENDEL, S.A. et al. The long face syndrome: vertical maxillary excess. Am J Orthod, St. Louis, v. 70, no. 4, p. 398-408, Oct. 1976.

69. SCHOTT, K: CAPELLI JÚNIOR, J. Estudo comparativo da análise de Tweed-Merrifield em brasileiros com oclusão excelente. Rev Soc Bras Ortodon, Rio de Janeiro, v. 3, n. 7, p. 291-297, jan./ jun. 1999.

70. SCHUDY, F. F. Vertical growth versus anteroposterior growth as related to function and treatment plane. Angle Orthod, Appleton, v.34, no.2, p.75-93, Apr. 1964.

71. SCHUDY, F. F. The rotation of the mandible resulting from growth: its implications in orthodontic treatment. Angle Orthod, Appleton, v. 35, no. 1, p. 36-50, Jan. 1965

72. SCHUDY, F. F. The Control of vertical overbite in clinical orthodontics. Angle Orthod, Appleton, v. 38, no. 1, p. 19-39, Jan. 1968.

73. SHUDY, G. F. Posttreatment craniofacial growth: its implications in orthodontic treatment. Am J Orthod, St. Louis, v. 65, no. 1, p. 39-57, Jan. 1974
74. SIRIWAT, P. P. JARABAK, J. R. Malocclusion and facial morphology: is there a relationship? Angle Orthod, Appleton, v. 55 no. 2, p. 127-138, Apr. 1985

75. SPYROPOULOS, M. N. ASKARIEH, M. Vertical control: a multifactorial problem and its clinical implications. Am J Orthod, St. Louis, v.70, no. 1, p. 70-80, July 1976

76. STEINER, C. Cephalometrics for you and me. Am J Orthod, St. Louis, v. 39, no. 10, p. 729-755, Oct. 1953.

77. STEINER, C. Cephalometrics in clinical practice. Angle Orthod Appleton, v. 29, no. 1, p. 8-29, Jan. 1959.

78. STEINER, C. The use of cephalometrics as an aid to planning and assessing orthodontic treatment. Am J Orthod, St. Louis, v. 46 , no. 10, p. $721-735$, Oct. 1960.

79. STEINER, C. Cephalometrics as a clinical tool. In: KRAUS, B. S. RIEDEL, R. A. Vistas in Orthodontics. Philadelphia: Lea \& Febinger, 1962. p.131-161.

80. SUBTELNY, J. D.; SAKUDA, M. Open-bite: diagnosis and treatment. Am J Orthod, St. Louis, v. 50, no. 5, p. 337-358, May 1964.

81. TOURNE, L. P. M. The long face syndrome and impairment of the nasopharyngeal airway. Angle Orthod, Appleton, v. 60, no. 3, p.167-176, Fall 1990.

82. TOUTOUNTZAKIS, N. E.; HARALABAKIS, N. B. A posteroanterior cephalometric evaluation of adult open bite subjects as related to normals. Eur J Orthod, Oxford, v.13, no. 5 , p. 410-415, Oct. 1991

83. TROUTEN, J. C. et al. Morphologic factors in open bite and deep bite. Angle Orthod, Appleton, v. 53, no. 3, p. 192-211, July 1983.

84. TWEED, C. H. Was the development of the diagnostic facial triangle as an accurate analysis based on fact or fancy? Am $\mathbf{J}$ Orthod, St. Louis, v. 48, no.11, p. 823-840, Nov. 1962.

85. URSI, W. J. S. et al. Sexual dimorphism in normal craniofacial growth. Angle Orthod, Appleton, v. 63, no. 1, p. 47-56, Spring 1993.

86. VADEN, J. L.; HARRIS, E. SINCLAIR, P. M. Clinical ramifications of posterior and anterior facial height changes between treated and untreated Class II samples. Am J Orthod Dentofacial Orthop, St. Louis, v. 105, no. 5, p. 438-443, May 1994.

87. VAN DER BEEK, M. C. J.; HOEKSMA, J. B.; ANDERSEN, P. Vertical facial growth: a longitudinal study from 7 to 14 years of age. Eur J Orthod, Oxford, v. 13, no. 3, p. 202-208, May 1991.

88. WEINBERG, H.; KRONMAN, J. H. Orthodontic influence upon anterior facial height. Angle Orthod, Appleton, v. 36, no. 1 p. 80-88, Jan. 1966.

89. WILLIAMS, B. H. Craniofacial proportionality in a horizontal and vertical plane, a study in norma lateralis. Angle Orthod, Appleton, v. 23, no. 1, p. 26-34, Jan. 1953.

90. WOODSIDE, D. G.; LINDER-ARONSON, S. The channelization of upper and lower anterior face heights compared to population standard in males between ages 6 to 20 years. Eur J Orthod, Oxford, v. 1, no. 1, p. 25-40, 1979

91. WYLIE, W. L. Overbite and vertical facial dimensions in terms of muscle balance. Angle Orthod, Appleton, v. 14, no.1/ 2, p.13-17, Jan./ Apr. 1944

92. WYLIE, W. L. The relatioship between ramus height, dental height, and overbite. Am J Orthod Oral Surg, St. Louis, v. 32, no. 2, p. 57-67, Feb. 1946

93. WYLIE, W. L. The assessment of anterior dysplasia. Angle Orthod, Appleton, v. 17, no. 3/4, p. 97-109, July/ Oct. 1947.

94. WYLIE, W. L.; JOHNSON, E. L. Rapid evaluation of facial dysplasia in the vertical plane. Angle Orthod, Appleton, v. 22, no. 3, p.165-182, July 1952.

\section{Endereço para correspondência}

Ricardo Takahashi

Disciplina de Ortodontia

Faculdade de Odontologia de Bauru/USP

Alameda Octávio Pinheiro Brizolla, 9-75 Caixa Postal 73.

Bauru/SP CEP 17043-101

E-mail: ricardotakahashi@aol.com 